\title{
ON THE FINAL FATE OF DIFFERENTIALLY ROTATING DEGENERATE CONFIGURATIONS
}

\author{
(Abstract)
}

\section{KOESTER}

Institute für Theoretische Physik und Sternwarte der Universität Kiel, F.R.G.

Double shell-burning stars of intermediate masses develop a degenerate carbon/oxygen core. Whether this core will ignite carbon depends on several parameters, i.e. mass, neutrino rates and rotation. If angular momentum is conserved, the core may even grow above the Chandrasekhar limit (Sackmann and Weidemann, 1972). In this case mass loss might terminate the nuclear evolution, leaving a remnant star which approximates a differentially rotating Ostriker-Bodenheimer (1968) configuration. The final fate of such configurations on cooling down is not yet clear.

Schwartz and Africk (1970) proposed that evolution leads to uniformly rotating cores with masses above the Chandrasekhar limit which finally end in collapse and supernova explosion. On the other hand Sackmann and Weidemann suggested rotational mass loss resulting in stable configurations below Chandrasekhar limit.

Recently, Durisen (1973) showed that viscosity does not in general lead to uniform rotation above the melting point of the ion lattice. For lower temperatures, however, the viscosity increases by several orders of magnitude such that the crystallized part of the star must certainly be in uniform rotation.

In order to follow the evolution during crystallization we made schematic model calculations, assuming always uniform rotation for the crystalline core and neglecting transport of angular momentum in the non-crystalline part of the model.

The configurations were assumed to be spherical with angular velocity depending only on the distance from the centre. The radial component of the centrifugal force is replaced by its mean value over the sphere. With these simplifications we constructed evolutionary sequences consisting of sequences of equilibrium models with constant angular momentum and mass but with growing crystalline cores. The results show that only if a differentially rotating model starts with very nearly the critical density may it undergo collapse by inverse $\beta$-decays (or ignite carbon). In most cases crystallization is terminated by mass loss, leaving as end products uniformly rotating degenerate stars below the Chandrasekhar (or James) limiting mass.

\section{References}

Ostriker, J. P. and Bodenheimer, P.: 1968, Astrophys. J. 151, 1089.

Sackmann, I. J. and Weidemann, V.: 1972, Astrophys. J. 178, 427.

Schwartz, R. and Africk, S.: 1970, Astrophys. Letters 5, 141.

Durisen, R. H.: 1973, Astrophys. J. 183, 205, 215. 